\title{
openheart Risk and outcomes of aortic valve endocarditis among patients with bicuspid and tricuspid aortic valves
}

\author{
Yuka Kiyota, ${ }^{1}$ Alessandro Della Corte, ${ }^{2}$ Vanessa Montiero Vieira, ${ }^{1}$ Karam Habchi, ${ }^{1}$ \\ Chuan-Chin Huang, ${ }^{1}$ Ester E Della Ratta, ${ }^{2}$ Thoralf M Sundt, ${ }^{3}$ Prem Shekar, ${ }^{4}$ \\ Jochen D Muehlschlegel, ${ }^{1}$ Simon C Body ${ }^{1}$
}

To cite: Kiyota Y, Della Corte A, Montiero Vieira V, et al. Risk and outcomes of aortic valve endocarditis among patients with bicuspid and tricuspid aortic valves. Open Heart 2017;4:e000545. doi:10.1136/ openhrt-2016-000545

Received 5 0ctober 2016 Revised 16 March 2017 Accepted 21 March 2017
CrossMark

${ }^{1}$ Department of Anesthesiology, Perioperative and Pain Medicine, Brigham and Women's Hospital, Boston, Massachusetts, USA

${ }^{2}$ Department of Cardiothoracic Sciences, Second University of Naples, V. Monaldi Hospital, Naples, Italy

${ }^{3}$ Department of Surgery, Massachusetts General Hospital, Boston, Massachusetts, USA ${ }^{4}$ Department of Surgery, Brigham and Women's Hospital, Boton, Massachusetts, USA

\section{Correspondence to}

Dr Simon C Body, Department of Anesthesiology, Perioperative and Pain Medicine, Brigham and Women's Hospital, 75 Francis St, Boston, MA 02115, USA; sbody@partners.org

\section{ABSTRACT}

Objective Patients with structural abnormalities of cardiac valves, including bicuspid aortic valve (BAV), are said to be at higher risk of infective endocarditis (IE). We sought to determine the risk of IE of the BAV compared with the tricuspid aortic valve (TAV) and to determine the risk of aortic valve replacement and mortality after IE. Methods From medical records of two US and one Italian hospitals, patients with their first episode of IE of any native valve were identified. In the US cohort 42 patients with BAV and 393 patients with TAV with IE occurring between 1 January 2000 and 30 June 2014 were identified. In the Italian cohort 48 patients with BAV and 341 patients with TAV with IE underwent valve replacement surgery between 1 January 2000 and1 November 2015. The risk of IE for BAV and TAV and subsequent outcomes were determined after matching to patients without IE.

Results After adjustment for risk factors, the risk of IE in the US cohort was 23.1 (95\% Cl 8.1 to $100, p<0.0001)$ times greater for BAV than TAV. Patients with BAV with IE were more likely to have an aortic root abscess. Within the subsequent 5 years, BAV patients with IE were more likely to undergo valve replacement (85\%) than TAV patients with IE (46\%). Patients with IE were at increased risk of death. The findings were similar in the Italian cohort.

Conclusions Patients with BAV are at markedly increased risk of IE and aortic root abscess than patients with TAV. Increased risk of IE in patients with BAV indicates they may be a candidate group for long-term trials of antibiotic prophylaxis of IE.

\section{INTRODUCTION}

Infective endocarditis (IE) is an uncommon but serious valvular heart disease with high morbidity and mortality. ${ }^{1}$ Patients with structural abnormalities of cardiac valves, including bicuspid aortic valve (BAV), are said to be at higher risk of $\mathrm{IE}^{2-5} \mathrm{BAV}$ is the most common congenital valvular heart disease among adults, with a prevalence of $0.5 \%-2 \%{ }^{6}$ ${ }^{7}$ and therefore patients with BAV constitute a significant proportion of patients with IE. As the prevalence of BAV in source populations is not precisely known, estimating the risk of IE in patients with $\mathrm{BAV}$, compared with

\section{KEY QUESTIONS}

What is already known about this subject?

- Infective endocarditis (IE) is an uncommon but serious valvular heart disease with high morbidity and mortality. Patients with structural abnormalities of cardiac valves, including bicuspid aortic valve (BAV), are said to be at higher risk of IE. Antibiotic prophylaxis of IE in patients with BAV and other congenital valve diseases is currently not recommended, but is being debated.

What does this study add?

- Our study finds that relative risk of IE of the BAV is markedly greater than for a tricuspid aortic valve (TAV). Patients with more comorbidities and lower income status were more likely to die within the following 5 years. Within the subsequent 5 years, patients with BAV with IE were more likely to have aortic root abscess and undergo valve replacement (85\%) than patients with TAV with IE (46\%).

How might this impact on clinical practice?

- Increased risk of IE in patients with BAV indicates they may be a candidate group for long-term trials of antibiotic prophylaxis of IE.

individuals with a tricuspid aortic valve (TAV) has been imprecise.

Antibiotic prophylaxis of IE in patients with BAV and other congenital valve diseases is currently not recommended, ${ }^{8}{ }^{9}$ but is being debated. ${ }^{1011}$ Although only very large randomised clinical trials of antibiotic prophylaxis will yield sufficient data to guide recommendations, ${ }^{12}$ it is known that individuals with structural heart disease are at increased risk of IE. However, the risk of IE in BAV, compared with TAV, is unknown and may indicate value of targeted antibiotic trials in this at-risk group.

We hypothesised that the risk of IE of the aortic valve would be higher for patients with a BAV than a TAV. We accounted for the unknown frequency of BAV in the source 
population using the frequency of non-aortic valve IE in both groups. The secondary hypothesis is that the occurrence of valve replacement and mortality after diagnosis of IE would be independent of aortic valve type (BAV vs TAV). Further, we wished to confirm a reported higher incidence of aortic root abscess in patients with BAV. ${ }^{13-15}$ We examined these hypotheses using the medical records of a US urban healthcare network and an Italian tertiary-referral university-affiliated hospital.

\section{METHODS}

\section{Patient populations}

We used the medical records from two cohorts. From the medical records of an urban Massachusetts healthcare network (Partners Healthcare,Boston, Massachusetts, USA) that encompasses approximately 3.94 million covered lives and with Institutional Review Board approval, we identified all adults ( $\geq 18$ years) with their first episode of native valve IE occurring between 1 January 2000 and 30 June 2014. Patients were identified by searches of International Classification of Diseases, Ninth Revision, Clinical Modification (ICD-9-CM) discharge diagnoses with the following codes: 421.0 (acute and subacute IE), 421.1 (endocarditis, valve unspecified, in diseases classified elsewhere), 421.9 (acute endocarditis,unspecified) and 424.9 (endocarditis, valve unspecified) yielding 46 patients with BAV and 472 TAV with IE. Text searches and individual review of discharge summaries, surgical records,microbiology reports, and transthoracic and transoesophageal echocardiogram reports were reviewed for diagnosis of BAV and IE. Patients with previous history of IE, previous valve replacement or repair, or congenital heart disease other than BAV were excluded from further analysis. Patients who met only the 'definite' modified Duke criteria of $\mathrm{IE}^{16}$ and received antibiotic treatment for IE were included in the analysis group of 41 patients with BAV IE and 371 patients with TAV IE. The date of IE diagnosis was defined as the first day of the echocardiogram findings consistent with endocarditis. The censoring date for surgical and mortality outcomes was 1 October 2015. Patients were followed for an average of $4.8 \pm 3.9$ years.

Two local Boston populations without IE were further identified for comparison of mortality outcomes. From a cohort of 4579 patients with BAV without history of IE obtained from the same source healthcare network, the 41 patients with BAV with IE were each matched to 11-38 control patients with BAV without IE for age \pm 10 years, gender and race, yielding matching to a population of 1081 patients with BAV without IE. Because the extent of functional degeneration of the BAV prior to IE was unavailable for the majority of patients with BAV, we did not use BAV structural abnormalities to match BAV IE cases with BAV IE controls. Patients with TAV with IE $(n=371)$ were matched on an up to $50: 1$ basis to TAV patients without IE for age \pm 10 years, gender, race and healthcare utilisation ${ }^{17}$ from the same source population who were not known to have a BAV, yielding a TAV control population of 18356 individuals matching on a 47-50:1 basis to 371 patients with TAV with IE. Two patients with TAV IE were unable to be matched. Sample size was determined by the number of patients with IE and an arbitrary cut-off of 50:1 control:case ratio.

Using a cohort of Italian patients who had undergone native valve replacement for IE we examined the frequency of outcomes after valve replacement from a single surgical centre's institutional database (V Monaldi Hospital, University of Naples, Naples, Italy). The source population of all patients with IE was not available so direct comparisons with the US population were not possible. Prospective collection and review of discharge summaries, surgical records, microbiology reports, and transthoracic and transoesophageal echocardiogram reports was performed for diagnosis of BAV and IE. Patients with previous history of IE, previous valve replacement or repair, or congenital heart disease other than BAV were excluded from further analysis. Patients who met 'definite' and 'possible' modified Duke criteria of $\mathrm{IE}^{16}$ and received surgery for IE between 1 January 2000 and 1 November 2015 were included in the analysis group of 48 patients with BAV and 341 patients with TAV. The date of IE diagnosis was defined as the first day of the echocardiogram findings consistent with endocarditis. The censoring date for surgical and mortality outcomes was 15 November 2015. Patients were followed for an average of 3.2 \pm 2.6 years after IE in the Italian cohort.

\section{Outcomes}

The primary hypothesis was to evaluate whether the incidence of aortic valve IE varies between patients with BAV and TAV. We implemented a case-control study design for this aim by first identifying 165 aortic valve-only IE cases among 412 patients with IE. The proportion of patients with BAV and TAV in the general population was not exactly known because a majority of patients with BAV is unaware of their BAV status until the occurrence of significant morbidity. Therefore, we used the patients with non-aortic valve IE as controls, assuming the proportions of patients with BAV/TAV are the same in patients with non-aortic valve IE and the general population, making the assumption that the incidence of IE of non-aortic valves would be the same between patients with BAV and TAV. We examined the incidence of aortic root abscess in patients with BAV by reviewing imaging records and operative reports. The secondary hypothesis was whether the risk of valve replacement and mortality after diagnosis of IE would be independent of aortic valve type. We examined this hypothesis using a combined outcome consisting of mortality and affected valve repair/replacement, whichever occurred first, after the first diagnosis of IE in BAV and TAV cohorts compared with valve-respective controls without IE.

Among US patients hospitalised with endocarditis, we determined the rates of 1-year, 5year and 10-year all-cause mortality from the US Social Security Death Index and 
local mortality resources, using the date of IE diagnosis for all mortality outcomes. For control patients without IE, we used the age of IE diagnosis of the matched patient with IE as beginning of the mortality observation period in the control patient. Overall mortality risk accounted for age, race, gender, decile of income estimated by postcode of residence ${ }^{1819}$ and comorbidity assessed by modified Elixhauser Score ${ }^{20}$ derived from ICD-9 codes occurring between 5 years and 30 days prior to the diagnosis of IE and calculated using an R package. ${ }^{21}$ For control patients, the modified Elixhauser Score was estimated using ICD-9 codes occurring between 5 years and 30 days prior to the age at the diagnosis of IE in their matched patients with IE. Comorbidities were calculated from ICD-9 codes using Elixhauser's mapping. ${ }^{22}{ }^{23}$ We also examined the frequency of valve surgery in IE cases and controls in the 30 days and 5 years after the diagnosis of IE. ${ }^{24}$

Among Italian patients who had undergone valve surgery for IE, mortality was examined by patient, family and physician contact at defined intervals. Complete follow-up was achieved for all patients. Income deciles and Elixhauser Scores were unavailable.

\section{Covariates}

Diabetes was defined as having type I or II diabetes, with or without drug treatment. Chronic obstructive pulmonary disease, cancer, lymphoma, renal and liver disease, obesity, alcohol and drug abuse, hypertension, congestive heart failure, stroke, rheumatic heart disease and arrhythmia were defined by diagnostic codes and medical records review. Finally, we examined medical records for reports of potential precipitating events for IE.

\section{Statistical methods}

Statistical analysis was performed with R V.3.1 (https:// www.r-project.org) and SAS V.9.4 (SAS Institute, Cary, North Carolina, USA). Categorical variables were summarised by frequency and analysed by $\chi^{2}$ and Fisher's exact tests. For the primary hypothesis, since the incidence of aortic-value IE was extremely low (less than 1:5000 person-years for BAV and lower for TAV), the OR estimated using a case-control study design would be a close approximation to the risk ratio. We interpreted the OR as risk ratios throughout this study. For the second hypothesis, Kaplan-Meier survival curves were compared using a two-sided non-parametrical log-rank test. Mortality analyses were conducted to assess the impact of valve type on short-term mortality and long-term mortality. Age was used as the time scale in the mortality analyses. For each exposure, we first performed a univariate analyses, followed by Cox proportional hazards models, adjusting for gender, race, disease severity and socioeconomic status. The proportional hazards assumption and assumption of linearity were tested using permutation testing based on martingale residuals. For all analyses, a two-sided $\mathrm{p}<0.05$ was considered statistically significant. The funding source had no role in the study design, analysis, interpretation or submission of the results.

\section{RESULTS}

From the US IE cohort we identified 435 patients with their first episode of native valve definite IE according to modified Duke criteria (table 1). The relative risk of IE of a BAV was 23.1 (95\% CI 8.1 to $100, \mathrm{p}<0.0001$ ) times greater than a TAV (table 2). This occurred despite patients with BAV being younger and having a lower overall frequency of comorbidities (table 1). The increased risk of IE in the BAV group was independent of patient age, with patients with BAV under 65 years of age having an IE relative risk of 15.6 (95\% CI 4.9 to 50 , $\mathrm{p}<0.0001)$. In patients with BAV, lower income decile and intravenous drug abuse were identified as independent predictors of IE. In patients with TAV, lower income decile, intravenous drug or alcohol abuse, previous valvular heart disease and other comorbidities were identified as independent predictors of IE. Age, gender and race were not used as predictors of IE, as they had been used in matching IE cases to controls. Patients with IE were more likely to undergo repair or replacement of the affected valve within 30 days and 1 year of their episode of IE, than population controls (table 1).

The Italian surgical cohort had a similar overall demographic, but with differences in prevalence of liver disease, hypertension and an implanted device (table 3). Like the US cohort, the risk of IE requiring surgery of the aortic valve, compared with other valves, was greater for BAV than TAV $(\mathrm{p}<0.0001)$ with all patients with BAV IE undergoing aortic valve replacement surgery compared with only $54.6 \%$ in the TAV IE cohort (table 4).

Unadjusted mortality was higher in patients with TAV IE than patients with BAV IE at the 1-year and 5-year observation points in the US cohort. After adjustment for age and the presence of a BAV, gender and race were not predictors of mortality (figure $1 \mathrm{~A}$ ). IE did not quite reach statistical significance as a univariate $(\mathrm{p}=0.059)$ or multivariate $(\mathrm{p}=0.052)$ predictor of mortality in patients with a BAV (figure 1B). However, age, race, Elixhauser Comorbidity Score and income decile were independent predictors of mortality (table 5).

IE was a significant univariate $(\mathrm{p}<0.001)$ and multivariate $(p<0.001)$ predictor of mortality in patients with a TAV (figure 2A). In addition, age, gender, race, Elixhauser Comorbidity Score and income decile were independent predictors of mortality (table 6). Based on an overall $\mathrm{BAV}$ prevalence of $1 \%$, we observed a raw IE case rate of one case per 134,048 patients with TAV and 12,658 patients with BAV per year.

In the Italian surgical cohort of patients undergoing valve repair or replacement for IE, mortality was higher in patients with TAV than patients with BAV at the 1-year and 5-year observations. Using Cox regression and after adjustment for age, the presence of a $\mathrm{BAV}(\mathrm{OR}=0.71$; $95 \%$ CI 0.22 to $2.3, \mathrm{p}=0.57$ ) was not an independent predictor of mortality (figure 2B).

We observed a higher incidence of aortic root abscess in patients with BAV compared with patients with TAV 
Table 1 Baseline characteristics of the US BAV and TAV IE and control populations

\begin{tabular}{|c|c|c|c|c|c|c|c|}
\hline & $\begin{array}{l}\text { BAV with IE } \\
n=41\end{array}$ & $\begin{array}{l}\text { BAV without } \\
\text { IE } n=1081\end{array}$ & $\begin{array}{l}\text { TAV with IE } \\
\mathrm{n}=371\end{array}$ & $\begin{array}{l}\text { TAV without } \\
\text { IE } n=18356\end{array}$ & $\begin{array}{l}\text { p value } \\
\text { BAV IE } \\
\text { versus } \\
\text { TAV IE }\end{array}$ & $\begin{array}{l}\text { p value } \\
\text { BAV IE } \\
\text { versus } \\
\text { BAV no IE }\end{array}$ & $\begin{array}{l}\text { p value } \\
\text { TAV IE } \\
\text { versus } \\
\text { TAV no IE }\end{array}$ \\
\hline \multicolumn{8}{|l|}{ Demographics } \\
\hline \multicolumn{8}{|l|}{ Age, years } \\
\hline$<40$ & $16(39 \%)$ & 297 (27\%) & $63(17 \%)$ & 3067 (17\%) & $<0.001$ & 0.71 & 0.98 \\
\hline $40-49$ & $10(24 \%)$ & $246(23 \%)$ & $50(13 \%)$ & $2272(12 \%)$ & & & \\
\hline $50-59$ & $5(12 \%)$ & 199 (18\%) & $63(17 \%)$ & 3117 (17\%) & & & \\
\hline $60-69$ & $7(17 \%)$ & $224(21 \%)$ & $84(23 \%)$ & $4313(23 \%)$ & & & \\
\hline $70-79$ & $2(5 \%)$ & $76(7 \%)$ & $76(20 \%)$ & $3690(20 . \%)$ & & & \\
\hline$>=80$ & $1(2 \%)$ & $39(4 \%)$ & $35(9 \%)$ & $1897(10 . \%)$ & & & \\
\hline Gender (female) & $31(76 \%)$ & $870(80 \%)$ & $232(63 \%)$ & $11424(62 \%)$ & 0.12 & 0.43 & 0.96 \\
\hline Race (Caucasian) & $36(88 \%)$ & 1026 (95\%) & $305(82 \%)$ & $15073(82 \%)$ & 0.51 & 0.063 & 1.0 \\
\hline \multicolumn{8}{|l|}{ Income decile } \\
\hline $0-4$ & $1(2 \%)$ & $55(5 \%)$ & $36(10 \%)$ & $1099(6 \%)$ & 0.058 & 0.045 & 0.004 \\
\hline $5-7$ & $12(29 \%)$ & $158(15 \%)$ & $66(19 \%)$ & 2827 (16\%) & & & \\
\hline $8-10$ & $27(66 \%)$ & $845(80 \%)$ & $251(71 \%)$ & $13583(78 \%)$ & & & \\
\hline \multicolumn{8}{|l|}{ Elixhauser Score ${ }^{\dagger}$} \\
\hline$<=0$ & $11(27 \%)$ & $187(17 \%)$ & $31(8 \%)$ & $3742(20 \%)$ & 0.047 & 0.45 & $<0.0001$ \\
\hline $1-10$ & $13(32 \%)$ & $389(36 \%)$ & $95(26 \%)$ & $5430(30 \%)$ & & & \\
\hline $11-20$ & $9(22 \%)$ & $296(27 \%)$ & $120(32 \%)$ & $3823(21 \%)$ & & & \\
\hline$>20$ & $7(17 \%)$ & $209(19 \%)$ & $122(33 \%)$ & $5361(29 \%)$ & & & \\
\hline Diabetes & $2(5 \%)$ & $125(12 \%)$ & $45(12 \%)$ & $2684(15 \%)$ & 0.20 & 0.22 & 0.15 \\
\hline COPD & $7(17 \%)$ & $219(20 \%)$ & $97(26 \%)$ & $6688(36 \%)$ & 0.19 & 0.70 & 0.0001 \\
\hline Cancer & $4(10 \%)$ & $135(12 \%)$ & $45(12 \%)$ & 4664 (25\%) & 0.80 & 0.81 & $<0.0001$ \\
\hline Lymphoma & $2(5 \%)$ & $35(3 \%)$ & $13(4 \%)$ & $960(5 \%)$ & 1.00 & 0.40 & 0.91 \\
\hline Renal disease & $6(15 \%)$ & $96(9 \%)$ & $115(31 \%)$ & $2850(16 \%)$ & 0.03 & 0.27 & $<0.0001$ \\
\hline Liver disease & $5(12 \%)$ & $123(11 \%)$ & $76(21 \%)$ & 3871 (21\%) & 0.22 & 0.81 & 0.90 \\
\hline Obesity & $5(12 \%)$ & $142(13 \%)$ & $25(7 \%)$ & $4199(23 \%)$ & 0.19 & 0.08 & 0.02 \\
\hline Alcohol abuse & $3(7 \%)$ & $58(5 \%)$ & $31(8 \%)$ & $1789(10 \%)$ & 1.00 & 0.48 & 0.0008 \\
\hline Drug abuse & $10(24 \%)$ & $61(6 \%)$ & $75(20 \%)$ & $1544(8 \%)$ & 0.42 & 0.0001 & $<0.0001$ \\
\hline \multicolumn{8}{|l|}{ Prior cardiac status } \\
\hline Hypertension & $15(37 \%)$ & $541(50 \%)$ & $178(48 \%)$ & $10697(58 \%)$ & 0.24 & 0.15 & 0.0002 \\
\hline Congestive heart failure & $18(44 \%)$ & $404(37 \%)$ & $204(55 \%)$ & $3923(21 \%)$ & 0.24 & 0.32 & $<0.0001$ \\
\hline Previous stroke & $10(25 \%)$ & $549(51 \%)$ & $61(17 \%)$ & $3329(18 \%)$ & 0.19 & 0.002 & 0.49 \\
\hline Rheumatic heart disease & $3(7 \%)$ & $41(4 \%)$ & $30(8 \%)$ & $1866(10 \%)$ & 1.0 & 0.20 & 0.22 \\
\hline Arrhythmia & $20(49 \%)$ & $603(56 \%)$ & $218(59 \%)$ & $8183(45 \%)$ & 0.31 & 0.52 & $<0.0001$ \\
\hline \multicolumn{8}{|l|}{ Valve disease } \\
\hline Aortic valve endocarditis & $38(93 \%)$ & - & $127(34 \%)$ & - & $<0.0001$ & - & - \\
\hline Mitral valve endocarditis & $8(20 \%)$ & - & $200(54 \%)$ & - & $<0.0001$ & - & - \\
\hline Tricuspid valve endocarditis & $1(2 \%)$ & - & $87(23 \%)$ & - & 0.0005 & - & - \\
\hline Pulmonary valve endocarditis & $0(0 \%)$ & - & $4(1 \%)$ & - & 1.0 & - & - \\
\hline Vegetation present & 40 (98\%) & - & $367(99 \%)$ & - & 0.40 & - & - \\
\hline Abscess present & $11(27 \%)$ & - & $15(4 \%)$ & - & $<0.0001$ & - & - \\
\hline \multicolumn{8}{|l|}{ Organism } \\
\hline Staphylococcus aureus & $8(20 \%)$ & - & $169(46 \%)$ & - & $0.016^{* \star}$ & - & - \\
\hline
\end{tabular}


Table 1 Continued

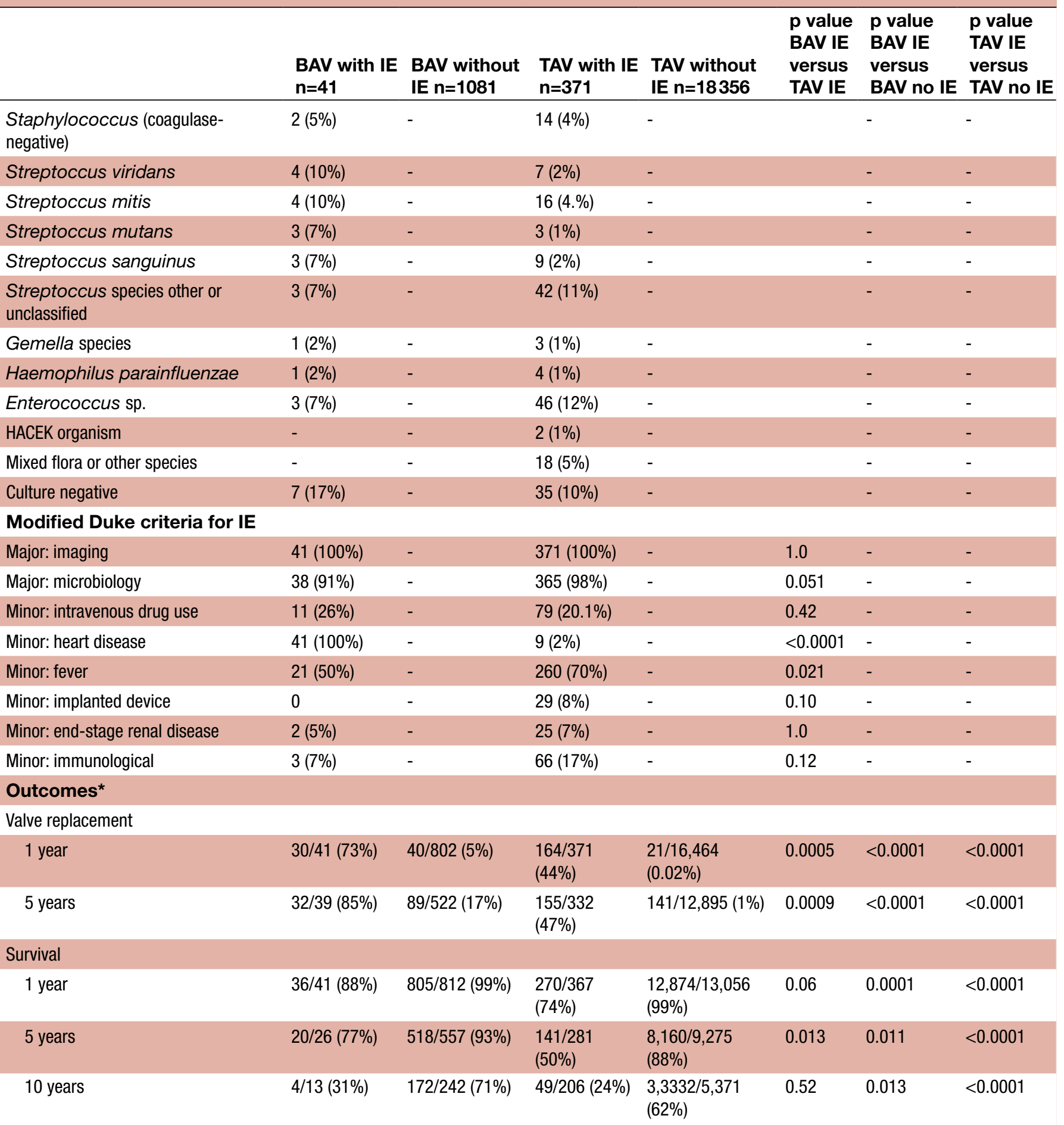

*After diagnosis of IE in the IE case, and the age-equivalent date in the non-IE control.

†Comparing patients with Staphylococcus aureus infection versus all other infections.

BAV, bicuspid aortic valve; COPD,Chronic Obstructive Pulmonary Disease; IE, infective endocarditis; TAV, tricuspid aortic valve; HACEK,

Haemophilus, Aggregatibacter, Cardiobacterium, Eikenella, Kingella.

in both the US (21\% vs $5 \%)$ and Italian cohorts $(25 \%$ vs $18 \%$ ). There was no evidence of a single organism being over-represented in patients with an aortic root abscess. Further, we observed a lower incidence of Staphylococcus aureus infection in patients with BAV with IE than patients with TAV with IE, in both the US (19\% vs 41\%) and Italian (8\% vs $17 \%)$ cohorts. Patients with BAV with IE were more likely to undergo valve replacement (85\%) than patients with TAV with IE $(46 \%)$, within the subsequent 5 years. Overall, patients with IE were much more likely to undergo valve replacement and were at increased risk of mortality than population controls. 
Table 2 Frequency of aortic valve infective endocarditis in patients with bicuspid and tricuspid aortic valves in the US cohort

\begin{tabular}{lccc}
\hline & Aortic valve-only infective endocarditis & Other valve infective endocarditis & Total \\
\hline Bicuspid aortic valve & $38(92.7 \%)$ & $3(7.3 \%)$ & 41 \\
Tricuspid aortic valve & $127(34.2 \%)$ & $244(65.8 \%)$ & 371 \\
\hline
\end{tabular}

Patients are classified as having IE of only the aortic valve, or having IE of any other valve including, or not including, the aortic valve. The observed risk ratio of having endocarditis of only the aortic valve was increased 23.1 times if the aortic valve was bicuspid (Cl 8.1 to 100$)$, $\mathrm{p}<0.0001$.

We did not observe a sufficient number of potentially precipitating events for IE and were unable to determine the rate of dental procedures or other events in the control populations. We therefore do not report these results.

\section{DISCUSSION}

Using patient records of a large urban Massachusetts healthcare network and a large Italian cohort of patients with IE, we observed that patients with a native BAV are at markedly increased risk of aortic valve endocarditis compared with endocarditis of other native heart valves. The relative risk of IE of a BAV was markedly greater than for a TAV in both study cohorts. As the prevalence of BAV in source populations is not precisely known and the majority of patients with BAV are unaware of their BAV status until the occurrence of significant morbidity, estimating the risk of IE in patients with BAV, compared with individuals with a TAV, has been imprecise. We therefore accounted for the unknown frequency of BAV in the source population using the frequency of non-aortic valve IE in both groups, making the assumption that the incidence of IE of non-aortic valves would be the same between patients with BAV and TAV. It is likely we observed a higher risk than recently reported ${ }^{25}$ by accounting for undiagnosed BAV in the community, by normalising the incidence of aortic valve IE and assuming equivalent risk of IE of other valves.

Older age and comorbidities measured by increased Elixhauser Score were significantly more frequent in patients with IE, independent of valve type. In the ageing population, there is a growing prevalence of patients with multiple comorbidities including end-stage renal disease and indwelling cardiac devices. ${ }^{26-28}$ In addition, there have been marked changes in the pattern of IE in the USA and the rest of the world, ${ }^{29}$ with reduced prevalence of rheumatic heart disease ${ }^{30}$ but an increased prevalence of intravenous drug use. ${ }^{31} 32$

The aetiology of BAV being associated with an increased frequency of IE is not established. It is can be hypothesised that altered flow patterns across the BAV may cause valvular endothelial injury, including platelet and fibrinogen deposition, that allows seeding of haematogenous bacteria ${ }^{33}$ and fungi. The wide variety of infecting organisms and absence of other evidence of immune deficiency does not favour a host-genome immunological defect in individuals with BAV. The observation that patients with BAV had a lower incidence of $S$. aureus infection than patients with TAV cannot yet be explained.

IE was a predictor of mortality in the US TAV population, but did not quite reach statistical significance in the BAV population, possibly because of the relatively small BAV IE population size and their younger age. S. aureus was the culprit organism in $38 \%$ of the patients, and is a known independent predictor for mortality. ${ }^{152} 274$ In concordance with prior literature, ${ }^{14}{ }^{15}$ patients with BAV with IE were much more likely to undergo valve replacement than their TAV counterparts and to have concurrent abscess of the aortic root.

\section{Study limitations}

This study has several limitations due to its retrospective nature. Data were not reliably present for possible IE-initiating events, including the dental hygiene of the patient and prior antibiotic prophylaxis for procedures. Further, the extent of aortic valve disease prior to the episode of IE could not be reliably found for the majority of patients. Thus we cannot offer an estimate of relative risk between a normally functioning aortic valve and a structurally or functionally abnormal aortic valve. Further, we do not provide information on the risk of IE of an artificial valve, as the risk of artificial valve IE is probably not dependent on the native valve.

This study does not provide guidance on the use of antibiotics in patients with BAV. However, we did observe a very high risk of IE in patients with BAV, thus establishing their potential value as a target population for randomised trials of prophylactic antibiotics in procedures prone to bacteraemia. The recent change in guidelines was largely motivated by the attempt to provide antibiotic prophylaxis to the highest-risk patients undergoing the highest-risk procedures. Although to this day, there is no randomised controlled study that tested the efficacy of antibiotic prophylaxis, BAV clearly increases the risk of IE in the otherwise healthy patient population. Our study suggests re-examination of congenital valvular heart disease among risk groups but does not provide any information regarding prophylactic administration of antibiotics prior to at-risk procedures. Patients with BAV should be encouraged to report symptoms of IE early to potentially reduce the risk of aortic root abscess. This study also highlights the risk of IE from intravenous drug use in patients with BAV. 
Table 3 Baseline characteristics of the Italian bicuspid aortic valve (BAV) and tricuspid aortic valve (TAV) infective endocarditis (IE) populations

\section{BAV with IE $n=48 \quad$ TAV with IE $n=341 \quad p$ value BAV IE versus TAV IE}

Demographics

Age, years

\begin{tabular}{|c|c|c|c|}
\hline$<40$ & $28(58.3 \%)$ & $55(16.1 \%)$ & \\
\hline $40-49$ & $9(18.8 \%)$ & $69(20.2 \%)$ & \\
\hline $50-59$ & $5(10.4 \%)$ & $82(24.1 \%)$ & $<0.0001$ \\
\hline $60-69$ & $6(12.5 \%)$ & $74(21.7 \%)$ & \\
\hline $70-79$ & 0 & $54(15.8 \%)$ & \\
\hline$>=80$ & 0 & $7(2.1 \%)$ & \\
\hline Gender (female) & $7(14.6 \%)$ & $100(29.3 \%)$ & 0.032 \\
\hline Race (Caucasian) & $48(100 \%)$ & $341(100 \%)$ & - \\
\hline Diabetes & $7(14.6 \%)$ & $41(12.0 \%)$ & 0.61 \\
\hline COPD & $3(6.3 \%)$ & $48(14.1 \%)$ & 0.13 \\
\hline Cancer & $1(2.1 \%)$ & $16(4.7 \%)$ & 0.41 \\
\hline Lymphoma & 0 & $2(0.6 \%)$ & 0.59 \\
\hline Renal disease & $4(8.3 \%)$ & $54(15.8 \%)$ & 0.17 \\
\hline Liver disease & $4(8.3 \%)$ & $81(23.8 \%)$ & 0.016 \\
\hline Obesity & $3(6.3 \%)$ & $13(3.8 \%)$ & 0.43 \\
\hline Drug abuse & $5(10.4 \%)$ & $56(16.4 \%)$ & 0.28 \\
\hline \multicolumn{4}{|l|}{ Prior cardiac status } \\
\hline Hypertension & $9(18.8 \%)$ & $181(53.1 \%)$ & $<0.0001$ \\
\hline Congestive heart failure & $8(16.7 \%)$ & $79(23.2 \%)$ & 0.31 \\
\hline Previous stroke & $5(10.4 \%)$ & $43(12.6 \%)$ & 0.67 \\
\hline Rheumatic heart disease & $3(6.7 \%)$ & $23(6.7 \%)$ & 0.90 \\
\hline Arrhythmia & $8(16.7 \%)$ & 65 (19.1\%) & 0.69 \\
\hline \multicolumn{4}{|l|}{ Valve disease } \\
\hline Aortic valve endocarditis & $48(100 \%)$ & $186(54.6 \%)$ & $<0.0001$ \\
\hline Mitral valve endocarditis & $10(20.8 \%)$ & $173(50.8 \%)$ & $<0.0001$ \\
\hline Tricuspid valve endocarditis & 0 & $53(15.3 \%)$ & $<0.0001$ \\
\hline Pulmonary valve endocarditis & 0 & $1(0.3 \%)$ & 0.71 \\
\hline Vegetation present & $41(85.4 \%)$ & $289(84.5 \%)$ & 0.90 \\
\hline Abscess present & $12(25.0 \%)$ & $60(17.6 \%)$ & 0.22 \\
\hline Organism (S. aureus) & $4(8.3 \%)$ & $57(16.7 \%)$ & 0.13 \\
\hline Valvular regurgitation & $48(100 \%)$ & $341(100 \%)$ & 1 \\
\hline \multicolumn{4}{|c|}{ Modified duke criteria for IE } \\
\hline Major: imaging & $41(85.4 \%)$ & $289(84.8 \%)$ & 0.90 \\
\hline Major: microbiology & $35(72.9 \%)$ & $225(66.0 \%)$ & 0.34 \\
\hline Minor: intravenous drug use & $5(10.4 \%)$ & $56(16.4 \%)$ & 0.28 \\
\hline Minor: heart disease & $48(100 \%)$ & $23(6.7 \%)$ & $<0.0001$ \\
\hline Minor: fever & $45(93.8 \%)$ & $280(82.1 \%)$ & 0.42 \\
\hline Minor: implanted device & $1(2.1 \%)$ & $44(12.9 \%)$ & 0.028 \\
\hline Minor: end-stage renal disease & $1(2.1 \%)$ & $21(6.2 \%)$ & 0.25 \\
\hline Minor: immunological & $3(6.7 \%)$ & $23(6.7 \%)$ & 0.90 \\
\hline \multicolumn{4}{|l|}{ Outcomes* } \\
\hline Survival & & & \\
\hline
\end{tabular}




\begin{tabular}{llll}
\hline Table 3 & Continued & & \\
\hline & BAV with IE $\mathbf{n = 4 8}$ & TAV with IE $\mathbf{n = 3 4 1}$ & p value BAV IE versus TAV IE \\
\hline 1 year & $41 / 44(93.2 \%)$ & $259 / 307(84.4 \%)$ & 0.12 \\
5 years & $13 / 16(81.2 \%)$ & $89 / 160(55.6 \%)$ & 0.048 \\
10 years & $0 / 3(0 \%)$ & $6 / 90(6.77 \%)$ & 0.64 \\
\hline
\end{tabular}

*After diagnosis of IE in the IE case, and the age-equivalent date in the non-IE control.

List of BAVCon Investigators and Sites

Eduardo Bossone, Azienda Ospedaliera Universitaria Salerno; Stefano Nistri, CMSR Veneto Medica, Vicenza; Dan Gilon, Hadassah-Hebrew University Medical Centre; Simon Body, Harvard University; Thoraf M. Sundt, Harvard University; J. Daniel Muehlschlegel, Harvard University; Alessandro Frigiola, IRCCS Policlinico San Donato; Francesca Pluchinotta, IRCCS Policlinico San Donato; Hector I. Michelena, Mayo Clinic; Maurice Sarano, Mayo Clinic; Bo Yang, Michigan University; Kim Eagle, Michigan University; Giuseppe Limongelli, Monaldi Hospital; Malenka Bissell, Oxford University; Alessandro DellaCorte, Second University of Naples; Gordon Huggins, Tufts University; Victor Dayan, Universidad de la República, Uruguay; Patrizio Lancelotti, Universite de Liege; Yohan Bossé, Université Laval; Patrick Mathieu, Université Laval; Evaldas Girdsaukas, University of Hamburg; Ashutosh Hardikar,
University of Hobart; Joseph Bavaria, University of Pennsylvania; Rita C. Milewski, University of Pennsylvania; Dianna M. Milewicz, University of Texas, Houston; Siddarth Prakash, University of Texas, Houston; Arturo Evangelista, Vall d'Hebron University Hospital; Joshua C Denny, Vanderbilt University; Edward Hulten, Walter Reed National Military Medical Centre.

Contributors All authors have contributed based on the following criteria: $s$ ubstantial contributions to the conception or design of the work; or the acquisition, analysis or interpretation of data for the work; $d$ rafting the work or revising it critically for important intellectual content; final approval of the version to be published; a greement to be accountable for all aspects of the work in ensuring that questions related to the accuracy or integrity of any part of the work are appropriately investigated and resolved.

Competing interests None declared.

Ethics approval This study protocol was approved by the institutional review boards of each site.

Provenance and peer review Not commissioned; internally peer reviewed.

Table 4 Frequency of aortic valve infective endocarditis in patients with bicuspid and tricuspid aortic valves in the Italian cohort

\begin{tabular}{lccc}
\hline & Aortic valve-only infective endocarditis & Other valve infective endocarditis & Total \\
\hline Bicuspid aortic valve & $38(79.2 \%)$ & $10(20.8 \%)$ & 48 \\
Tricuspid aortic valve & $115(33.7 \%)$ & $226(66.3 \%)$ & 371 \\
\hline
\end{tabular}

Patients are classified as having IE of only the aortic valve, or having IE of any other valve including, or not including, the aortic valve. The observed risk ratio of having endocarditis of only the aortic valve was increased 7.5 times if the aortic valve was bicuspid (Cl 3.6 to 15.5$)$, $\mathrm{p}<0.0001$.
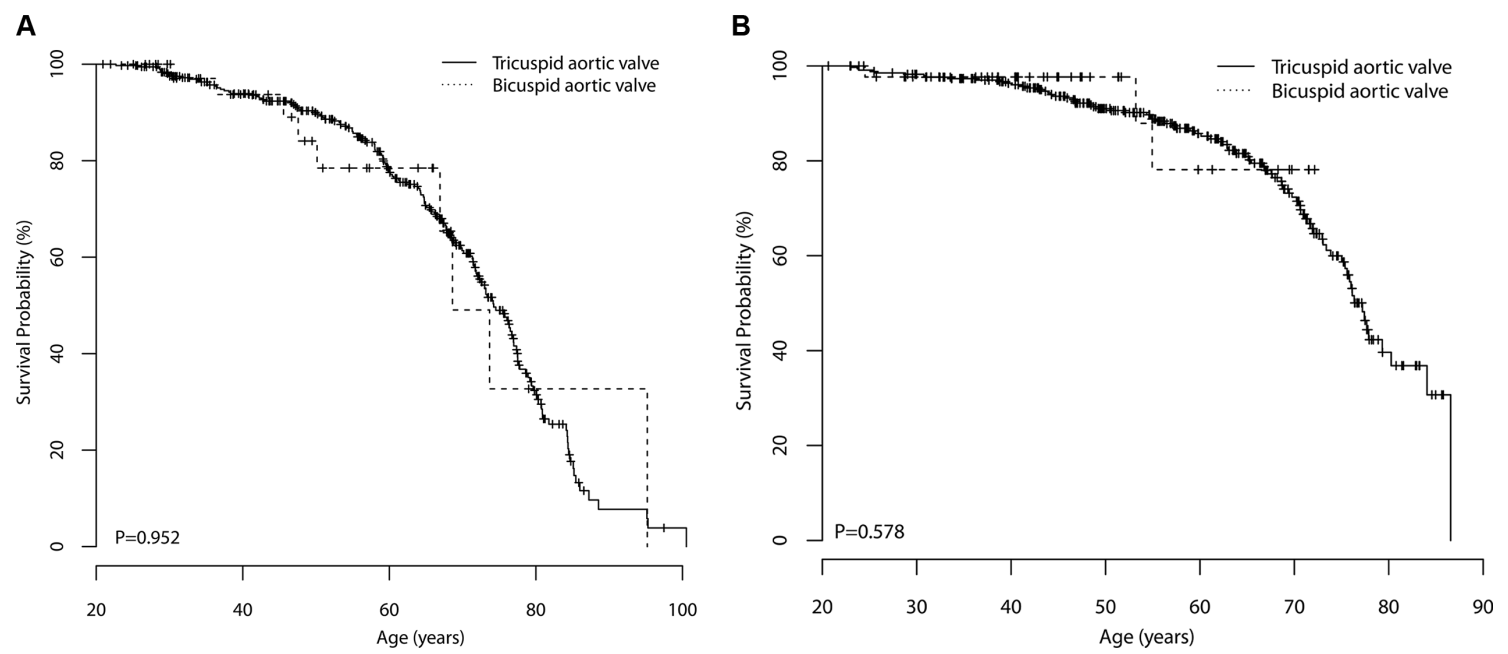

Figure 1 Comparison of survival in patients with BAV and TAV with IE in the US (A) and Italian (B) cohorts using Kaplan-Meier curves stratified by valve type. Patients with BAV IE had similiar sevival to patients wit TAV IE. The $p$ values from log rank tests comparing the two Kaplan Meier curves are shown. Censoring is indicated by tick marks on the curves. BAV, bicuspid aortic valve; IE, infective endocarditis; TAV, tricuspid aortic valve. 
Table 5 Univariate and multivariate predictors of mortality for US patients with infective endocarditis

\begin{tabular}{|c|c|c|c|c|}
\hline \multirow[b]{2}{*}{ Variables } & \multicolumn{2}{|l|}{ Univariate } & \multicolumn{2}{|l|}{ Multivariate } \\
\hline & HR (95\% Cl) & p Value & HR $(95 \% \mathrm{Cl})$ & p Value \\
\hline \multicolumn{5}{|c|}{ Bicuspid aortic valve } \\
\hline Yes & 0.98 (0.50 to 1.93$)$ & 0.95 & 1.00 (0.47 to 1.98$)$ & 0.98 \\
\hline \multicolumn{5}{|l|}{ Gender } \\
\hline Female & Ref & & Ref & \\
\hline \multicolumn{5}{|l|}{ Race } \\
\hline Caucasian & Ref & & Ref & \\
\hline Not Caucasian & 1.00 (0.67 to 1.50$)$ & 1.00 & 0.88 (0.58 to 1.33$)$ & 0.54 \\
\hline Elixhauser Score ${ }^{\star}$ & $1.02(1.01$ to 1.03$)$ & 0.003 & 1.02 (1.00 to 1.03$)$ & \\
\hline Income decile* & 0.93 (0.88 to 0.99$)$ & 0.023 & 0.94 (0.89 to 1.00$)$ & 0.07 \\
\hline
\end{tabular}

*Defined as a one point or decile increment

Open Access This is an Open Access article distributed in accordance with the Creative Commons Attribution Non Commercial (CC BY-NC 4.0) license, which permits others to distribute, remix, adapt, build upon this work non-commercially, and license their derivative works on different terms, provided the original work is properly cited and the use is non-commercial. See: http://creativecommons.org/ licenses/by-nc/4.0/

(C) Article author(s) (or their employer(s) unless otherwise stated in the text of the article) 2017. All rights reserved. No commercial use is permitted unless otherwise expressly granted.

\section{REFERENCES}

1. Bikdeli B, Wang Y, Kim N, et al. Trends in hospitalization rates and outcomes of endocarditis among medicare beneficiaries. J Am Coll Cardiol 2013;62:2217-26.

2. Li L, Wang H, Wang L, et al. Changing profile of infective endocarditis: a clinicopathologic study of 220 patients in a single medical center from 1998 through 2009. Tex Heart Inst $J$ 2014;41:491-8.

3. Lewis T, Grant R. Observations relating to subacute infective endocarditis. Heart 1923;4:21-99.

4. Sandre RM, Shafran SD. Infective endocarditis: review of 135 cases over 9 years. Clin Infect Dis 1996;22:276-86.
5. Choudhury R, Grover A, Varma J, et al. Active infective endocarditis observed in an indian hospital 1981-1991. Am J Cardiol 1992;70:1453-8.

6. Prakash SK, Bossé Y, Muehlschlegel JD, et al. A roadmap to investigate the genetic basis of bicuspid aortic valve and its complications: insights from the International BAVCon (Bicuspid aortic valve consortium). J Am Coll Cardiol 2014;64:832-9.

7. Michelena HI, Prakash SK, Della Corte A, et al. Bicuspid aortic valve: identifying knowledge gaps and rising to the challenge from the international bicuspid aortic valve consortium (BAVCon). Circulation 2014;129:2691-704.

8. Wilson W, Taubert KA, Gewitz M, et al. Prevention of infective endocarditis: guidelines from the american heart association: a guideline from the american heart association rheumatic fever, endocarditis, and kawasaki disease committee, council on cardiovascular disease in the young, and the council on clinical cardiology, council on cardiovascular surgery and anesthesia, and the quality of care and outcomes research interdisciplinary working group. Circulation 2007;116:1736-54.

9. National Institute for Health and Clinical Excellence. Prophylaxis against infective endocarditis 2008. (NICE clinical guideline No. 64 , 2008. http://www.nice.org.uk/CG064.

10. Dayer MJ, Jones S, Prendergast B, et al. Infective endocarditis and antibiotic prophylaxis - Authors' reply. Lancet 2015;386:531-2.
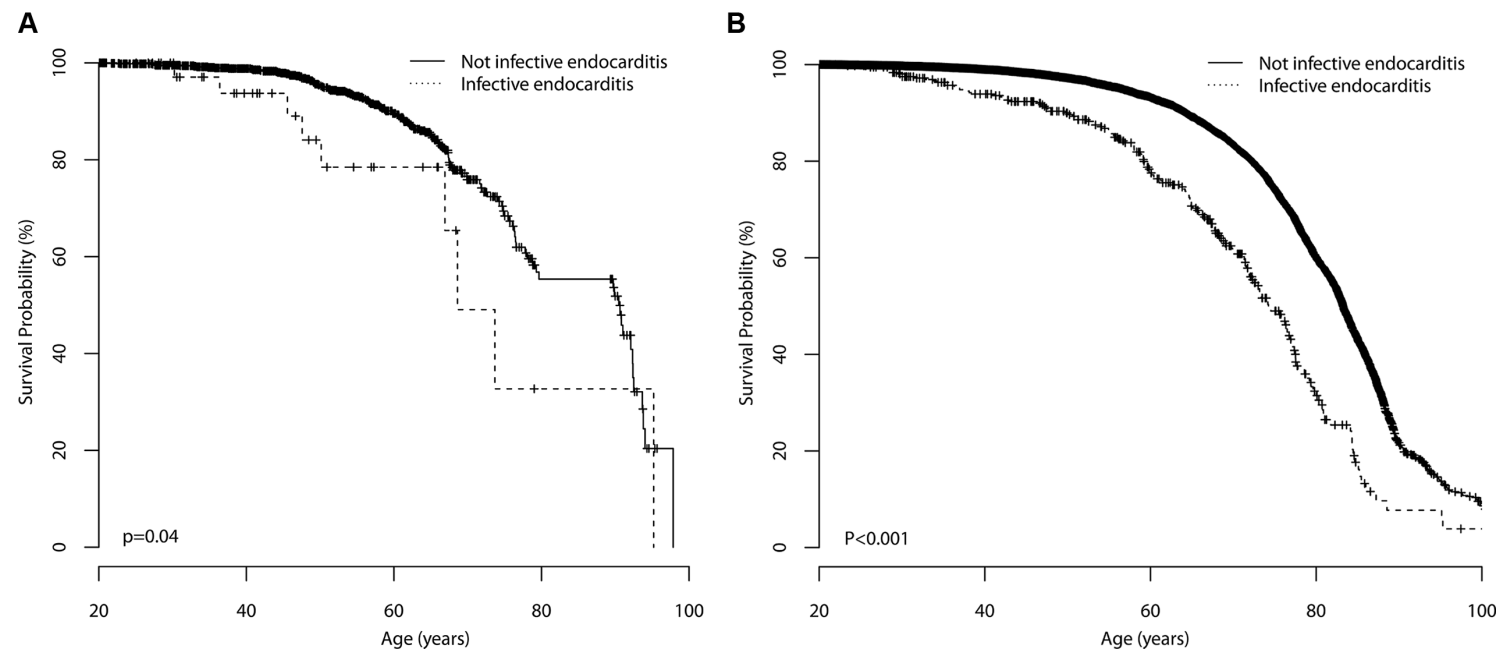

Figure 2 Comparison of survival in patients with BAV (A) and TAV (B) in the US cohort using Kaplan-Meier curves, stratified by whether or not they had suffered from IE. Patients with TAV with IE had lower survival compared with patients with TAV who had not suffered from IE, derived from $p$ values from log-rank tests. Patients with BAV with IE did not have lower survival ccompared with patients with BAV who had not suffered from IE. Censoring is indicated by tick marks on the curves. BAV, bicuspid aortic valve; IE, infective endocarditis; TAV, tricuspid aortic valve. 


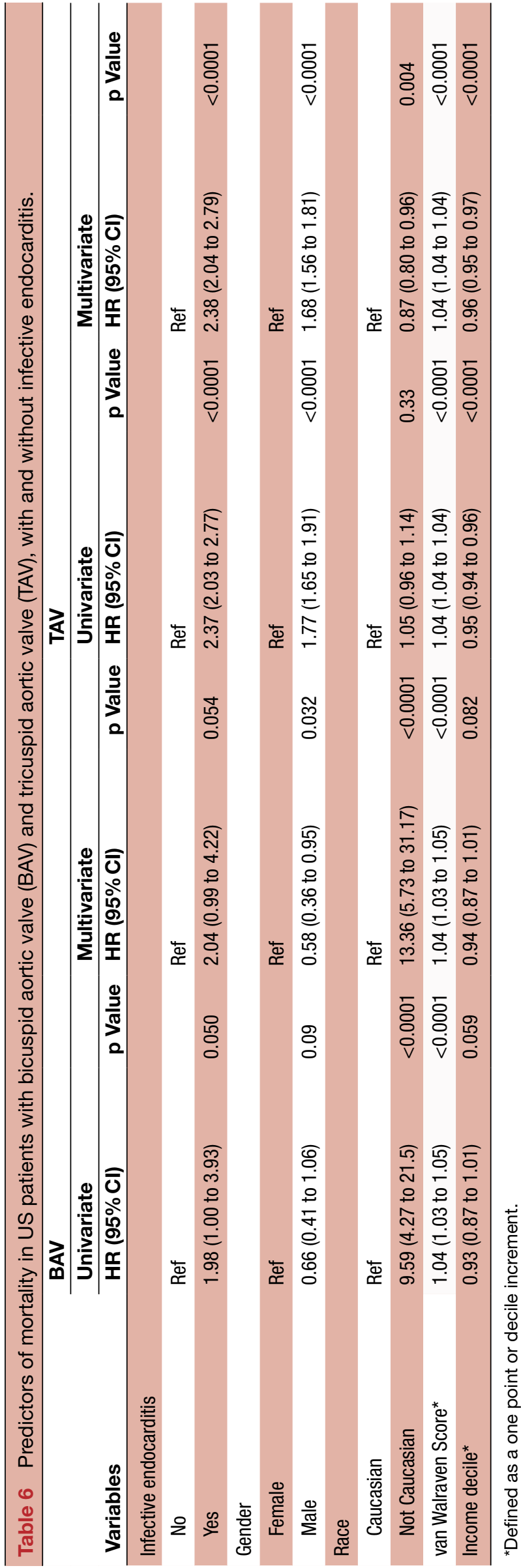

11. Dayer MJ, Jones $\mathrm{S}$, Prendergast $\mathrm{B}$, et al. Incidence of infective endocarditis in England, 2000-13: a secular trend, interrupted timeseries analysis. Lancet 2015;385:1219-28.

12. Duval $X$, Hoen B. Prophylaxis for infective endocarditis: let's end the debate. Lancet 2015;385:1164-5.

13. Kahveci G, Bayrak F, Pala S, et al. Impact of bicuspid aortic valve on complications and death in infective endocarditis of native aortic valves. Tex Heart Inst J 2009;36:111-6.

14. Lamas CC, Eykyn SJ. Bicuspid aortic valve--A silent danger: analysis of 50 cases of infective endocarditis. Clin Infect Dis 2000;30:336-41.

15. Tribouilloy C, Rusinaru D, Sorel C, et al. Clinical characteristics and outcome of infective endocarditis in adults with bicuspid aortic valves: a multicentre observational study. Heart 2010;96:1723-9.

16. Li JS, Sexton DJ, Mick N, et al. Proposed modifications to the Duke criteria for the diagnosis of infective endocarditis. Clin Infect Dis 2000;30:633-8

17. Castro VM, Apperson WK, Gainer VS, et al. Evaluation of matched control algorithms in EHR-based phenotyping studies: a case study of inflammatory bowel disease comorbidities. J Biomed Inform 2014:52:105-11.

18. Kim C, Diez Roux AV, Hofer TP, et al. Area socioeconomic status and mortality after coronary artery bypass graft surgery: the role of hospital volume. Am Heart J 2007:154:385-90.

19. Hanchate AD, Schwamm LH, Huang W, et al. Comparison of ischemic stroke outcomes and patient and hospital characteristics by race/ethnicity and socioeconomic status. Stroke 2013;44:469-76.

20. van Walraven C, Austin PC, Jennings A, et al. A modification of the Elixhauser comorbidity measures into a point system for hospital death using administrative data. Med Care 2009;47:626-33.

21. Wasey J. Tools for working with ICD-9 codes and Finding Comorbidities. 2015 https://github.com/jackwasey/icd9.

22. Elixhauser A, Steiner C, Harris DR, et al. Comorbidity measures for use with administrative data. Med Care 1998;36:8-27.

23. Sharabiani MT, Aylin P, Bottle A. Systematic review of comorbidity indices for administrative data. Med Care 2012;50:1109-18.

24. Aranki SF, Adams DH, Rizzo RJ, et al. Determinants of early mortality and late survival in mitral valve endocarditis. Circulation 1995;92(9 Suppl):143-9.

25. Michelena HI, Katan O, Suri RM, et al. Incidence of infective endocarditis in patients with bicuspid aortic valves in the community Mayo Clin Proc 2016;91:122-3.

26. Cecchi E, Chirillo F, Castiglione A, et al. Clinical epidemiology in italian registry of infective endocarditis (RIEI): Focus on age, intravascular devices and enterococci. Int J Cardiol 2015;190:151-6.

27. Martínez-Sellés M, Muñoz P, Arnáiz A, et al. Valve surgery in active infective endocarditis: a simple score to predict in-hospital prognosis. Int J Cardiol 2014:175:133-7.

28. Lu KJ, Kearney LG, Ord M, et al. Age adjusted Charlson Comorbidity index is an independent predictor of mortality over long-term follow-up in infective endocarditis. Int $J$ Cardiol 2013;168:5243-8.

29. Murdoch DR, Corey GR, Hoen B, et al. Clinical presentation, etiology, and outcome of infective endocarditis in the 21st century: the international collaboration on Endocarditis-Prospective cohort study. Arch Intern Med 2009;169:463-73.

30. Cahill TJ, Prendergast BD. Infective endocarditis. Lancet 2016;387:882-93.

31. Tung MK, Light M, Giri R, et al. Evolving epidemiology of injecting drug use-associated infective endocarditis: a regional centre experience. Drug Alcohol Rev 2015;34:412-7.

32. Pang PY, Sin YK, Lim CH, et al. Surgical management of infective endocarditis: an analysis of early and late outcomes. Eur $\mathrm{J}$ Cardiothorac 2015;47:826-32.

33. Veloso TR, Chaouch A, Roger T, et al. Use of a human-like low-grade bacteremia model of experimental endocarditis to study the role of staphylococcus aureus adhesins and platelet aggregation in early endocarditis. Infect Immun 2013;81:697-703.

34 Ohara T, Nakatani S, Kokubo Y, et al. Clinical predictors of inhospital death and early surgery for infective endocarditis: results of CArdiac disease REgistration (CADRE), a nation-wide survey in japan. Int J Cardiol 2013;167:2688-94. 